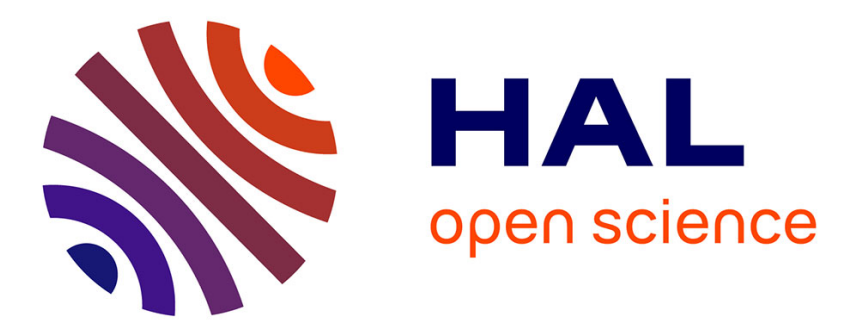

\title{
Quantum dot spontaneous emission control in a ridge waveguide
}

Petr Stepanov, Adrien Delga, Xiaorun Zang, Joël Bleuse, Emmanuel Dupuy, Emanuel Peinke, Philippe Lalanne, Jean-Michel Gérard, Julien Claudon

\section{- To cite this version:}

Petr Stepanov, Adrien Delga, Xiaorun Zang, Joël Bleuse, Emmanuel Dupuy, et al.. Quantum dot spontaneous emission control in a ridge waveguide. Applied Physics Letters, 2015, 106, pp.41112 121108. 10.1063/1.4906921 . hal-01381358

\section{HAL Id: hal-01381358 \\ https://hal-iogs.archives-ouvertes.fr/hal-01381358}

Submitted on 14 Oct 2016

HAL is a multi-disciplinary open access archive for the deposit and dissemination of scientific research documents, whether they are published or not. The documents may come from teaching and research institutions in France or abroad, or from public or private research centers.
L'archive ouverte pluridisciplinaire HAL, est destinée au dépôt et à la diffusion de documents scientifiques de niveau recherche, publiés ou non, émanant des établissements d'enseignement et de recherche français ou étrangers, des laboratoires publics ou privés. 


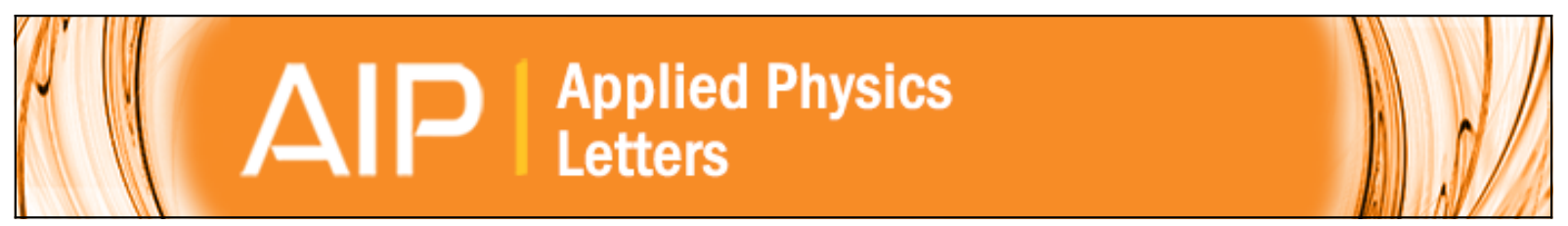

\section{Quantum dot spontaneous emission control in a ridge waveguide}

Petr Stepanov, Adrien Delga, Xiaorun Zang, Joël Bleuse, Emmanuel Dupuy, Emanuel Peinke, Philippe Lalanne, Jean-Michel Gérard, and Julien Claudon

Citation: Applied Physics Letters 106, 041112 (2015); doi: 10.1063/1.4906921

View online: http://dx.doi.org/10.1063/1.4906921

View Table of Contents: http://scitation.aip.org/content/aip/journal/apl/106/4?ver=pdfcov

Published by the AIP Publishing

\section{Articles you may be interested in}

Broadband Purcell enhanced emission dynamics of quantum dots in linear photonic crystal waveguides

J. Appl. Phys. 112, 093520 (2012); 10.1063/1.4764923

Spontaneous emission control of single quantum dots in bottom-up nanowire waveguides

Appl. Phys. Lett. 100, 121106 (2012); 10.1063/1.3694935

Slow-light-enhanced single quantum dot emission in a unidirectional photonic crystal waveguide

Appl. Phys. Lett. 96, 031109 (2010); 10.1063/1.3294298

The impact of thermal effects on emission characteristics of asymmetrical AIGAO-waveguide microdisks based on quantum dots

Appl. Phys. Lett. 91, 121108 (2007); 10.1063/1.2785127

Spontaneous emission mechanisms of $\mathrm{Ga}$ In As N/Ga As quantum dot systems

J. Appl. Phys. 100, 124311 (2006); 10.1063/1.2401051

\section{Pure Metals - Ceramics} Alloys $•$ Polymers in dozens of forms
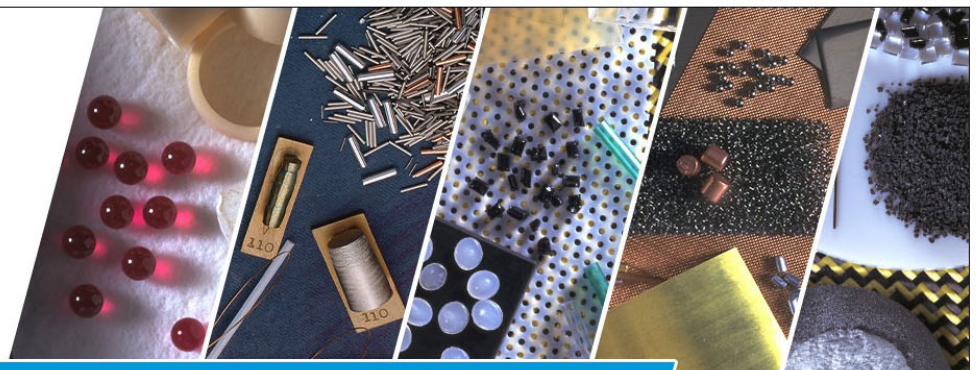

Small quantities fast Expert technical assistance $5 \%$ discount on online orders

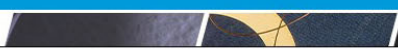




\title{
Quantum dot spontaneous emission control in a ridge waveguide
}

\author{
Petr Stepanov, ${ }^{1}$ Adrien Delga, ${ }^{1}$ Xiaorun Zang, ${ }^{2}$ Joël Bleuse, ${ }^{1}$ Emmanuel Dupuy, ${ }^{1}$ \\ Emanuel Peinke, ${ }^{1}$ Philippe Lalanne, ${ }^{2}$ Jean-Michel Gérard, ${ }^{1}$ and Julien Claudon ${ }^{1, a)}$ \\ ${ }^{1}$ CEA-CNRS-UJF group "Nanophysique et Semiconducteurs," CEA, INAC, SP2M, F-38054 Grenoble, France \\ ${ }^{2}$ Laboratoire Photonique, Numérique et Nanosciences, Institut d'Optique d'Aquitaine, Univ. Bordeaux, CNRS, \\ 33405 Talence, Cedex, France
}

(Received 10 November 2014; accepted 17 January 2015; published online 30 January 2015)

\begin{abstract}
We investigate the spontaneous emission (SE) of self-assembled InAs quantum dots (QDs) embedded in GaAs ridge waveguides that lay on a low index substrate. In thin enough waveguides, the coupling to the fundamental guided mode is vanishingly small. A pronounced anisotropy in the coupling to non-guided modes is then directly evidenced by normal-incidence photoluminescence polarization measurements. In this regime, a measurement of the QD decay rate reveals a SE inhibition by a factor up to 4. In larger wires, which ensure an optimal transverse confinement of the fundamental guided mode, the decay rate approaches the bulk value. Building on the good agreement with theoretical predictions, we infer from calculations the fraction $\beta$ of SE coupled to the fundamental guided mode for some important QD excitonic complexes. For a charged exciton (isotropic in plane optical dipole), $\beta$ reaches 0.61 at maximum for an on-axis QD. In the case of a purely transverse linear optical dipole, $\beta$ increases up to 0.91 . This optimal configuration is achievable through the selective excitation of one of the bright neutral excitons. (C) 2015 AIP Publishing LLC.
\end{abstract}

[http://dx.doi.org/10.1063/1.4906921]

Semiconductor optical waveguides that embed individual quantum dots (QDs) feature appealing assets to realize integrated quantum photonic circuits for quantum information processing or quantum simulation. ${ }^{1}$ In most implementations, achieving a large coupling between the emitter and a single guided mode is of critical importance. In this perspective, the fraction $\beta$ of spontaneous emission (SE) funnelled into the guided mode of interest constitutes a very important figure of merit. Nowadays, state-of-the-art photonic crystal waveguides offer close to unity $\beta$-factors. ${ }^{2-4}$ However, the large transmission losses of this waveguide technology ${ }^{5}$ prevents a straightforward scaling up to large photonic chips. One way to get around this difficulty is to couple a short photonic crystal section to a ridge waveguide, ${ }^{6}$ which features excellent optical transmission. ${ }^{7}$ Alternatively, in a much simpler approach, the whole photonic circuit-including the QD section - can be built with ridge waveguides. Using this strategy, an on-chip QD-beam splitter and QD-spin interface have been recently demonstrated. ${ }^{8,9}$ On the detection side, a superconducting nanowire located in the evanescent field of a ridge waveguide can be used to detect guided photons with low noise and high efficiency. ${ }^{10}$ Despite these promising advances, the tailoring of QD spontaneous emission by ridge waveguides has not yet been investigated experimentally.

We address here this important question for individual self-assembled InAs QDs integrated in GaAs ridge waveguides that lay on a low index planar substrate. This geometry, amenable to large scale integration, is inspired by a seminal work on quantum well microlasers. ${ }^{11}$ By combining polarization-resolved measurements and luminescence time decay analysis, we provide a comprehensive experimental investigation of the QD SE dynamics. Experimental results closely follow theoretical predictions, and we build on this

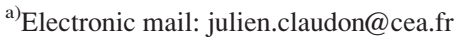

agreement to discuss theoretically the $\beta$-factor for important QD excitonic complexes. In particular, we predict that the selective excitation of a purely transverse linear optical dipole leads to $\beta$ up to 0.91 .

The ridge waveguide under consideration is sketched in Fig. 1(a). It is made of GaAs, a material with a large refractive index $(n=3.45)$ and lays on a low index substrate made of $\mathrm{Si}_{3} \mathrm{~N}_{4}(n=1.99)$, silica $(n=1.45)$, and epoxy $(n \approx 1.5)$. Its rectangular section features a fixed height $h=130 \mathrm{~nm}$ and a width $w$. The spatial profile of the fundamental guided mode $\mathrm{M}$ is shown in Fig. 1(b). A linear optical dipole with a freespace wavelength $\lambda=950 \mathrm{~nm}$ is located on the photonic wire axis. The dipole can take two in plane orientations: longitudinal $(\|)$ or transverse $(\perp)$. Figure 1(c) shows the calculated SE rate into $\mathrm{M}\left(\Gamma_{\mathrm{M}}\right)$ and the total SE rate $(\Gamma)$ for both dipole configurations. All rates are normalized to the bulk value $\Gamma_{\text {bulk }}$. The calculation was performed using a fully vectorial modal method based on Fourier-series expansion in the transverse directions. ${ }^{12,13}$ Both the coupling to $\mathrm{M}$ and the coupling to free-space modes are strongly dependent on the dipole orientation. For a transverse dipole, an optimal coupling to $\mathrm{M}$ is achieved for $w / \lambda=0.26$, yielding $\Gamma_{\mathrm{M} \perp} / \Gamma_{\text {bulk }}=0.98$. Moreover, the coupling to free-space modes is strongly inhibited, a consequence of a dielectric screening phenomenon. ${ }^{14}$ In contrast, one always has $\Gamma_{\mathrm{M} \|}=0$ on the wire axis. The total SE rate then directly accounts for the coupling to freespace modes, and Fig. 1(c) shows that dielectric screening is absent in the longitudinal dipole configuration. As discussed in the end of the letter, this photonic anisotropy makes the fraction of SE funneled into M highly dependent on the nature and orientation of the emitter dipole.

We now use self-assembled InAs QDs as a probe of the photonic environment defined by the ridge waveguide. As sketched in Fig. 1(a), the dots are integrated in the central plane of the GaAs wire. The sample is not intentionally 

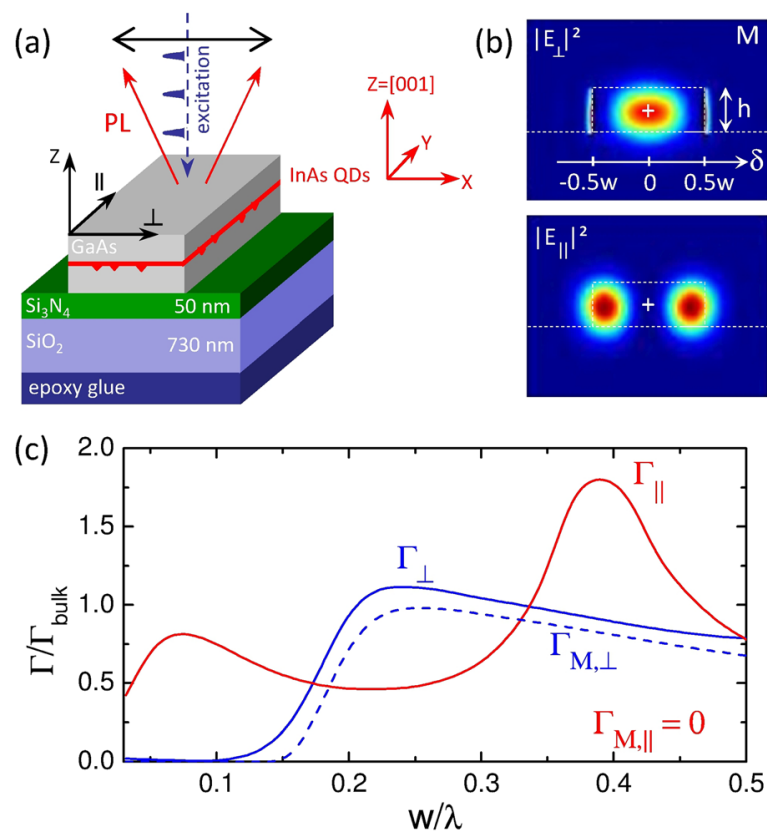

FIG. 1. (a) Sketch of the QD-ridge waveguide system and geometry of the optics experiments. The $(X, Y, Z)$ axis system corresponds to QD crystalline eigenaxes and $(\perp, \|)$ to the photonic eigenaxes. (b) Spatial intensity profile of the fundamental guided mode $\mathrm{M}$ calculated for $h=130 \mathrm{~nm}, w=300 \mathrm{~nm}$, and $\lambda=950 \mathrm{~nm} . E_{\|}\left(E_{\perp}\right)$ is the longitudinal (transverse) electric field component associated with $\mathrm{M}$. (c) Calculated normalized SE rates plotted against the reduced wire width $w / \lambda$ for linear optical dipoles, oriented along $\|$ and $\perp$. The wire is infinitely long and the dipole is located on the wire axis $(\delta=0)$.

doped and the QDs were grown along the $Z=[001]$ direction. Within a few degrees of accuracy, the photonic axes $\|$ and $\perp$ are parallel to the crystal axes $X=[110]$ and $Y=[1 \overline{1} 0]$, which are easy cleavage axis of GaAs. ${ }^{15}$ The fabrication process consists of four main steps: (1) the growth of a planar structure in a molecular beam epitaxy chamber, (2) the deposition of the $\mathrm{Si}_{3} \mathrm{~N}_{4}$ and $\mathrm{SiO}_{2}$ layers, (3) a flipchip step in which the structure is transferred on an host substrate, and (4) the top-down definition of waveguides. We have fabricated series of $30 \mu \mathrm{m}$ long ridge waveguides with a nominal $w$ ranging from $100 \mathrm{~nm}$ to $500 \mathrm{~nm}$. The actual widths have been measured with a $10 \mathrm{~nm}$ accuracy using SEM imaging.

The optical properties of individual QDs located in ridge waveguides are investigated in a cryogenic $(4 \mathrm{~K})$ microphotoluminescence ( $\mu \mathrm{PL}$ ) setup. As schematized in Fig. 1(a), excitation and collection are performed along the $Z$ direction. Pulsed excitation is provided by a Ti-Sa laser tuned to $840 \mathrm{~nm}$, in the absorption continuum of the QD wetting layer. The laser beam is focused on the sample with a microscope objective $(\mathrm{NA}=0.75)$, which also collects the sample luminescence. After a low pass filter that blocks the residual laser light, the luminescence is spectrally dispersed with a grating spectrometer equipped with a CCD camera. For a low excitation power, the $\mu \mathrm{PL}$ spectra (not shown) consist of spectrally isolated, sharp lines corresponding to recombination from neutral or charged QD excitons. Polarization- and time-resolved measurements are conducted using a rotating $\lambda / 2$ wave plate and a fixed linear polarizer located in front of the spectrometer and a silicon avalanche photodiode. The polarization analysis angle is measured with respect to the sample direction $(X)$.

We first consider individual QDs embedded in "thin" wires $(w / \lambda \approx 0.12)$, for which the coupling to guided modes is vanishingly small [Fig. 1(c)]: the radiative recombination dynamics is then dominated by the coupling to free-space modes. As exemplified in Fig. 2(a), the decay dynamics is then significantly slower than the one of dots embedded in a close-to-bulk photonic environment (curve "ref"). This reference was obtained on a QD ensemble located in the center of a large square ( $20 \mu \mathrm{m}$ side), patterned on the same sample, close to the wires of interest. Both time traces are well described by a single exponential decay, before reaching a constant level, associated with the detector dark counts. For the QD investigated in Fig. 2(a), the decay rate is $\Gamma=0.23 \mathrm{~ns}^{-1}$, which corresponds to a SE inhibition factor of 4.3 with respect to the bulk reference $\left(\Gamma_{\text {bulk }}=1 \mathrm{~ns}^{-1}\right){ }^{16}$ Moreover, the measured decay rate is independent on the polarization analysis angle. In addition, Fig. 2(b) shows that the emission of QDs in "thin" wires features a pronounced (a)

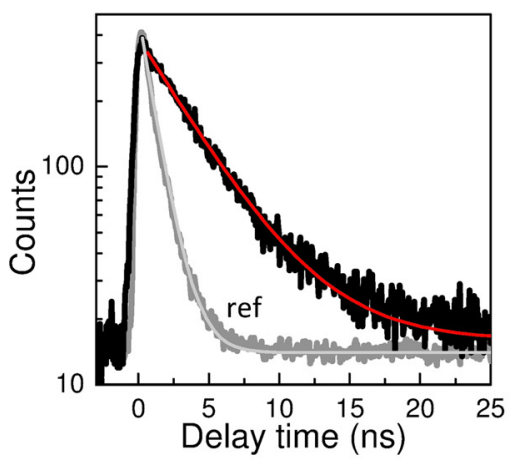

(b)

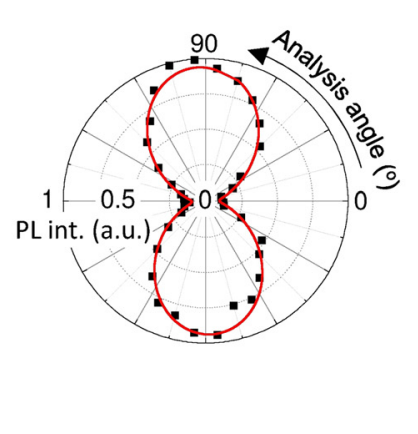

(c)

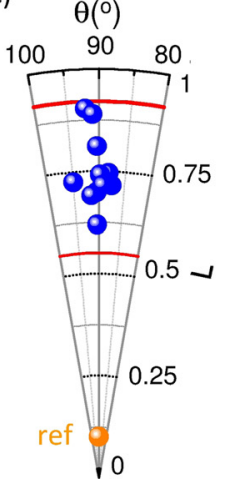

(d)

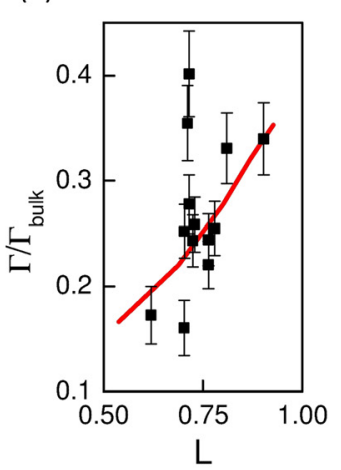

FIG. 2. Optical properties of individual QDs embedded in "thin" wires ( $w / \lambda=0.12)$, for which the coupling to guided modes is negligible. (a) Time-resolved luminescence decay (vertical log scale). The curve "ref" corresponds to an ensemble of QDs embedded in a close to bulk photonic environment ( $20 \mu \mathrm{m}$ large square pattern). The solid lines are fit to a mono-exponential decay, including a constant background. (b) Typical polarization analysis. (c) Polarization angle $\theta$ and degree of linear polarization $L$ measured for several QDs embedded in wires with similar dimensions. The point "ref" corresponds to an ensemble of QDs located close to the center of the large square pattern. The solid-red lines correspond to lower and upper bounds for $L$, calculated when the lateral position of the emitter is varied from $\delta / w=0.49$ (close to the sidewall) to $\delta / w=0$ (on-axis emitter). (d) Normalized SE rates plotted against $L$. The squares correspond to experiments and the solid line to theory. We have selected here QDs whose decay rate falls between the theoretical bounds of Fig. 3 . 
linear polarization, aligned along $\|$. As shown in Fig. 2(c), the degree of linear polarization $L$ varies from dot to dot from 0.6 to 0.9 . This polarization is imposed by the photonic structure: the ensemble of QDs located in the reference square pattern exhibits indeed a much smaller average linear polarization with $L \approx 0.1$ [point "ref" in Fig. 2(c)].

To interpret these results, we first note that a "thin" wire constitutes a very anisotropic photonic environment $\left(\Gamma_{\perp} \ll \Gamma_{\|}\right)$. Nevertheless, for the vast majority of QDs studied in this work, we reproducibly obtain single exponential decays, with a rate that do not depend on the polarization analysis angle. Following Ref. 17, we conclude that the emitter is simultaneously coupled to the $\|$ and $\perp$ decay channels with a single decay rate that can be calculated as the average $\Gamma=\frac{1}{2}\left(\Gamma_{\|}+\Gamma_{\perp}\right)$. In the present configuration, only the transverse dipole component experiences a pronounced SE inhibition [Fig. 1(c)], which leads to $\Gamma \approx \Gamma_{\|} / 2$. In contrast, for thin wires defined along the dot growth axis, all dipole components simultaneously experience a strong SE inhibition and inhibition factors that exceed ten have been reported. ${ }^{14,18}$ The polarization analysis sheds a complementary light on the anisotropy in the coupling to free-space modes. Indeed, when the emitter is simultaneously coupled to $\|$ and $\perp$ free-space modes, a fraction $\Gamma_{\|} /\left(\Gamma_{\|}+\Gamma_{\perp}\right)$ of SE is preferentially emitted in $\|$-polarized modes. If the differences in collection efficiencies for transverse and longitudinal polarizations are negligible, the degree of linear polarization can be expressed as $L=\left|\Gamma_{\|}-\Gamma_{\perp}\right| /\left(\Gamma_{\|}+\Gamma_{\perp}\right)$ (the coupling to $\mathrm{M}$ is vanishingly small). This simple analysis yields a calculated minimum $L=0.54$ for an off-axis emitter $(\delta / w=0.49)$ and a maximum value $L=0.93$ for $\delta / w=0$. As shown in Fig. 2(c), the experimental data fall well within these bounds; the scatter is associated with variations of the dot lateral position $\delta$. As a final consistency check, Fig. 2(d) shows normalized SE rates plotted against $L$ for QDs embedded in thin wires $(w / \lambda=0.12)$. The experimental data follow the predicted trend, which is plotted as a solid line. Such observations are compatible with the radiative recombination of a charged exciton (isotropic in plane optical dipole). They could also correspond to the recombination of a neutral exciton, provided that an additional spinflip mechanism couples the two bright excitons on a timescale shorter than radiative lifetimes. ${ }^{19}$

Figure 3 presents normalized decay rates for 120 individual QD lines in waveguides with $w / \lambda$ in the range of $0.1-0.3$. In particular, thicker wires $(w / \lambda=0.27)$ provide a tight lateral confinement of the fundamental guided mode $\mathrm{M}$ [Fig. 1(c)]. The emission rate can be then as high as $\Gamma=0.84 \mathrm{~ns}^{-1}$, a signature of a good coupling to $\mathrm{M}$. The two solid curves in Fig. 3 are the calculated maximum and minimum rate in an infinitely long wire, obtained by evaluating the average $\frac{1}{2}\left(\Gamma_{\|}+\Gamma_{\perp}\right)$ for various lateral positions $\delta$ of the emitter. The minimum always corresponds to a QD located close to the waveguide sidewall. The maximum coincides most of the time with an on-axis emitter (dashed trace). Within experimental errors, the data remain confined within the theoretical bounds. For thin wires, some rare dots exhibit a decay rate that exceeds the radiative limit, likely because of inevitable non-radiative processes associated with a close proximity to wire sidewalls.

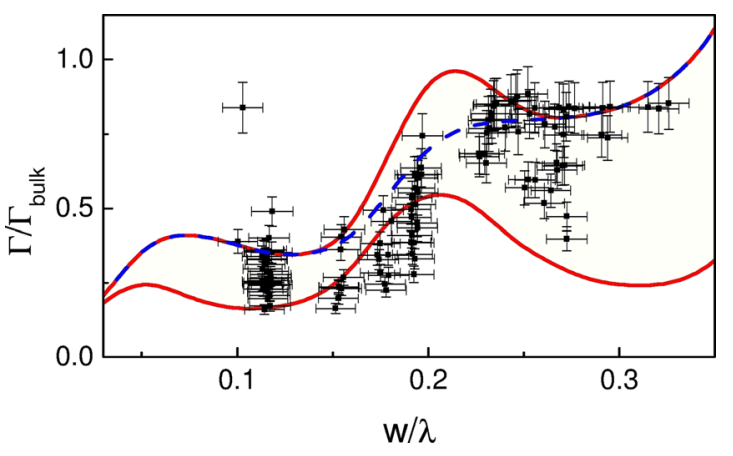

FIG. 3. Normalized SE rate of $\sim 120$ excitonic lines embedded in wires with various $w / \lambda$. The solid lines are the calculated maximum and minimum SE rates, obtained by varying the lateral position of the emitter from $\delta / w=0$ to 0.49 . The blue-dashed line corresponds to $\delta / w=0$. The calculation assumes an infinitely long wire.

We now theoretically evaluate the fraction $\beta$ of SE coupled to $\mathrm{M}$ for two important QD excitonic complexes. For a charged exciton (isotropic in-plane optical dipole), one has $\beta_{\text {iso }}=\left(\Gamma_{\mathrm{M} \|}+\Gamma_{\mathrm{M} \perp}\right) /\left(\Gamma_{\|}+\Gamma_{\perp}\right)$. This quantity, which includes coupling to forward and backward modes, is plotted against $w / \lambda$ in Fig. 4(a). For an on-axis emitter, the maximum value is achieved for $w / \lambda=0.24$, leading to $\beta_{\text {iso }}=0.61$. To evaluate the tolerance to QD misalignment, the same quantity is also plotted for two lateral positions $\delta / w=0.25$ and 0.49. As discussed in Ref. 17, the case of the neutral exciton is more subtle. A typical (asymmetric) neutral InAs QD features two bright excitonic transitions, detuned by a few $\mu \mathrm{eV}$ to a few tens of $\mu \mathrm{eV}$, with linear optical dipoles aligned along the $[110]$ and $[1 \overline{1} 0]$ crystal directions. In the presence of a fast spin-flip between the two bright states, $\beta=\beta_{\text {iso }}$. Without spin-flip, $\beta$ depends on the orientation of the photonic eigenaxes relative to the QD eigenaxes. A $45^{\circ}$ angle leads again to $\beta=\beta_{\text {iso. If the waveguide is aligned }}$ along [110] or [110], one should consider separately the transverse and longitudinal optical dipole, which feature $\beta_{\perp}=\Gamma_{\mathrm{M} \perp} / \Gamma_{\perp}$ and $\beta_{\|}=\Gamma_{\mathrm{M} \|} / \Gamma_{\|}$, respectively. If both dipoles are equally excited, $\beta$ is the average $\frac{1}{2}\left(\beta_{\|}+\beta_{\perp}\right)$. Interestingly, the selective excitation of the transverse dipole

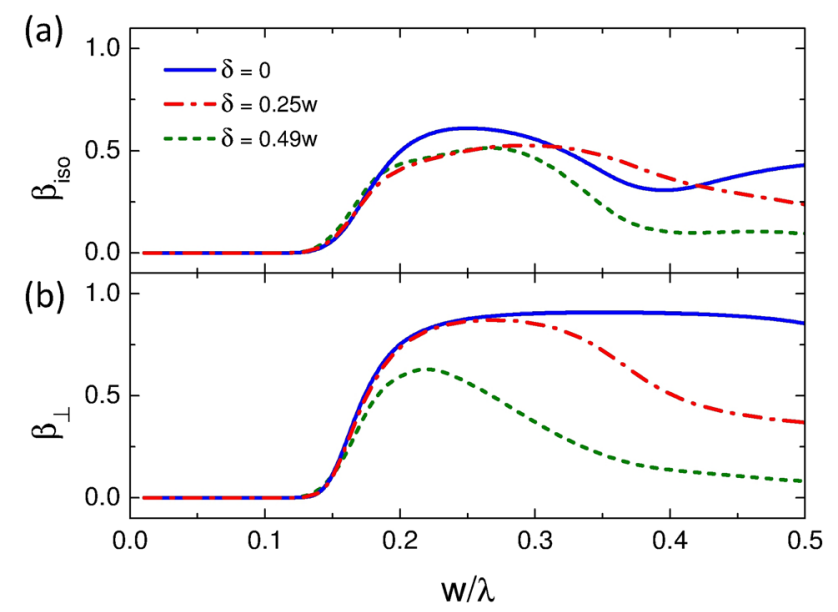

FIG. 4. Calculated $\beta$-factor for two emitter dipole configurations: (a) corresponds to an in-plane, isotropic emitter, and (b) to a transverse, linear optical dipole. The associated $\beta$-factors, respectively, $\beta_{\text {iso }}$ and $\beta_{\perp}$, are plotted against $w / \lambda$. In both cases, three traces are shown, corresponding to $\delta / w=0$ (on-axis), 0.25 , and 0.49 (on the sidewall). The wire is infinitely long. 
(e.g., using strictly resonant pumping ${ }^{20}$ ) optimizes SE control by the waveguide (in particular, the suppression of the coupling to free-space modes). As illustrated in Fig. 4(b), $\beta$ can be as large as 0.91 for a transverse optical dipole located on the axis of a waveguide with $w / \lambda=0.36$.

In a broader context, the present results highlight the importance of the optical dipole orientation to optimize SE control in a dielectric photonic wire. Such considerations are also particularly relevant for out-of-plane nanowire antennas, which have been developed to realize bright sources of quantum light for free-space applications. ${ }^{21-23}$ The first wire antennas were initially etched along the growth axis of selfassembled InAs QDs, ensuring an optimal transverse orientation for QD dipoles. ${ }^{24,25}$ Since then, InP nanowire antennas embedding an InAsP QD have also been obtained by direct growth. ${ }^{26}$ This approach ensures that a single emitter is located exactly on the wire axis, but also potentially changes the nature of the QD dipole. In the InAsP/InP system, a pronounced SE inhibition was observed in thin wires ${ }^{18}$ strongly supporting a transverse dipole orientation. ${ }^{14}$ Interestingly enough, II-VI QDs defined in thin nanowire display a fast decay dynamics. ${ }^{27}$ This behavior could be explained by a longitudinal optical dipole component associated with a-at least partial-light hole character of the exciton.

In conclusion, we have realized planar photonic wires and investigated in detail the SE dynamics of self-assembled QDs inserted in such structures. Calculations and experiments highlight the crucial impact of the optical dipole polarizations on the fraction of SE funneled into the guided mode. This figure-of-merit can approach unity, provided that a transverse linear dipole is selectively excited for an on-axis emitter. Beyond on-chip generation of single photons, such robust structures can also be exploited to explore singlephoton non-linearities or to mediate a photonic coupling between spatially separated emitters.

The authors acknowledge the support of the European Union Seventh Framework Program 209 (FP7/2007-2013) under Grant Agreement No. 601126210 (HANAS). X.Z. acknowledges a fellowship from Univ. Bordeaux. Sample fabrication was carried out in the "Plateforme Technologique Amont (PTA)" and CEA LETI MINATEC/DOPT clean rooms.

${ }^{1}$ P. Lodahl, S. Mahmoodian, and S. Stobbe, e-print arXiv:1312.1079.

${ }^{2}$ T. Lund-Hansen, S. Stobbe, B. Julsgaard, H. Thyrrestrup, T. Sünner, M. Kamp, A. Forchel, and P. Lodahl, Phys. Rev. Lett. 101, 113903 (2008).

${ }^{3}$ A. Schwagmann, S. Kalliakos, I. Farrer, J. P. Griffiths, G. A. C. Jones, D. A. Ritchie, and A. J. Shields, Appl. Phys. Lett. 99, 261108 (2011).
${ }^{4}$ M. Arcari, I. Söllner, A. Javadi, S. Lindskov Hansen, S. Mahmoodian, J. Liu, H. Thyrrestrup, E. Lee, J. Song, S. Stobbe, and P. Lodahl, Phys. Rev. Lett. 113, 093603 (2014).

${ }^{5}$ S. J. McNab, N. Moll, and Y. A. Vlasov, Opt. Express 11, 2927 (2003).

${ }^{6}$ S. Fattah Poor, T. B. Hoang, L. Midolo, C. P. Dietrich, L. H. Li, E. H. Linfield, J. F. P. Schouwenberg, T. Xia, F. M. Pagliano, F. W. M. van Otten, and A. Fiore, Appl. Phys. Lett. 102, 131105 (2013).

${ }^{7}$ I. Hiroaki, K. Hiruma, K. Ishida, T. Asai, and H. Matsumura, IEEE Trans. Electron Devices 32, 2662 (1985).

${ }^{8}$ N. Prtljaga, R. J. Coles, J. OHara, B. Royall, E. Clarke, A. M. Fox, and M. S. Skolnick, Appl. Phys. Lett. 104, 231107 (2014).

${ }^{9}$ I. J. Luxmoore, N. A. Wasley, A. J. Ramsay, A. C. T. Thijssen, R. Oulton, M. Hugues, S. Kasture, V. G. Achanta, A. M. Fox, and M. S. Skolnick, Phys. Rev. Lett. 110, 037402 (2013).

${ }^{10}$ J. P. Sprengers, A. Gaggero, D. Sahin, S. Jahanmirinejad, G. Frucci, F. Mattioli, R. Leoni, J. Beetz, M. Lermer, M. Kamp, S. Höfling, R. Sanjines, and A. Fiore, Appl. Phys. Lett. 99, 181110 (2011).

${ }^{11}$ J. P. Zhang, D. Y. Chu, S. L. Wu, S. T. Ho, W. G. Bi, C. W. Tu, and R. C. Tiberio, Phys. Rev. Lett. 75, 2678 (1995).

${ }^{12}$ G. Lecamp, J. P. Hugonin, and P. Lalanne, Opt. Express. 15, 11042 (2007).

${ }^{13}$ For the sake of simplification, all simulations are performed by assuming that the refractive indices of the epoxy glue is equal to that of $\mathrm{SiO}_{2}$. This approximation does not impact the following discussion.

${ }^{14}$ J. Bleuse, J. Claudon, M. Creasey, N. S. Malik, J.-M. Gérard, I. Maksymov, J.-P. Hugonin, and P. Lalanne, Phys. Rev. Lett. 106, 103601 (2011).

${ }^{15}$ We have not determined if $X=[110]$ or [110]. However, this ambiguity does not impact the results discussed in the paper.

${ }^{16}$ The QD ensemble located in the square pattern exhibits a decay rate $\Gamma_{\text {square }}=0.91 \mathrm{~ns}^{-1}$. A theoretical evaluation of the photonic density of states experienced by an in-plane dipole embedded in the planar structure yields $\Gamma_{\text {square }}=0.9 \times \Gamma_{\text {bulk }}$ leading to a bulk decay rate $\Gamma_{\text {bulk }}=1 \mathrm{~ns}^{-1}$.

${ }^{17}$ M. Munsch, J. Claudon, J. Bleuse, N. S. Malik, E. Dupuy, J.-M. Gérard, Y. Chen, N. Gregersen, and J. Mørk, Phys. Rev. Lett. 108, 077405 (2012).

${ }^{18}$ G. Bulgarini, M. E. Reimer, T. Zehender, M. Hocevar, E. P. A. M. Bakkers, L. P. Kouwenhoven, and V. Zwiller, Appl. Phys. Lett. 100, 121106 (2012).

${ }^{19}$ I. Favero, G. Cassabois, C. Voisin, C. Delalande, P. Roussignol, R. Ferreira, C. Couteau, J. P. Poizat, and J. M. Gérard, Phys. Rev. B 71, 233304 (2005).

${ }^{20}$ M. N. Makhonin, J. E. Dixon, R. J. Coles, B. Royall, E. Clarke, M. S. Skolnick, and A. M. Fox, e-print arXiv:1404.3967.

${ }^{21}$ I. Friedler, C. Sauvan, J. P. Hugonin, P. Lalanne, J. Claudon, and J. M. Gérard, Opt. Express 17, 2095 (2009).

${ }^{22}$ G. Grzela, R. Paniagua-Domnguez, T. Barten, Y. Fontana, J. A. SánchezGil, and J. Gómez Rivas, Nano Lett. 12, 5481 (2012).

${ }^{23}$ J. Claudon, N. Gregersen, P. Lalanne, and J.-M. Gérard, ChemPhysChem 14, 2393 (2013).

${ }^{24}$ J. Claudon, J. Bleuse, N. S. Malik, M. Bazin, P. Jaffrennou, N. Gregersen, C. Sauvan, P. Lalanne, and J.-M. Gérard, Nat. Photonics 4, 174 (2010).

${ }^{25}$ M. Munsch, N. S. Malik, J. Bleuse, E. Dupuy, A. Delga, J.-M. Gérard, J. Claudon, N. Gregersen, and J. Mørk, Phys. Rev. Lett. 110, 177402 (2013).

${ }^{26}$ M. E. Reimer, G. Bulgarini, N. Akopian, M. Hocevar, M. B. Bavinck, M. A. Verheijen, E. P. A. M. Bakkers, L. P. Kouwenhoven, and V. Zwiller, Nat. Commun. 3, 737 (2012).

${ }^{27}$ A. Tribu, G. Sallen, T. Aichele, R. André, J.-P. Poizat, C. Bougerol, S. Tatarenko, and K. Kheng, Nano Lett. 8, 4326 (2008). 